\title{
Teneurs en coumarines de 15 ligneux fourragers du Burkina Faso
}

\author{
Sibiri SOULAMA ${ }^{1 *}$, Odile Germaine NACOULMA ${ }^{2}$, Roland NAGTIERO MEDA ${ }^{2}$, \\ Joseph Issaka BOUSSIM ${ }^{3}$ et Jeanne MILLOGO-RASOLODIMBY ${ }^{3}$
}

\author{
${ }^{1}$ Département Productions Forestières, INERA, CNRST 03 BP 476 Ouagadougou 03, Burkina Faso. \\ ${ }^{2}$ Laboratoire de Biochimie et Chimie Appliquées (LABIOCA), Unité de Formation et de Recherche en Sciences \\ de la Vie et de la Terre, Université de Ouagadougou, 03 BP 7021 Ouagadougou 03, Burkina Faso. \\ ${ }^{3}$ Laboratoire de Biologie et Ecologie Végétales, Unité de Formation et de Recherche en Sciences de la Vie et \\ de la Terre, Université de Ouagadougou, 03 BP 7021 Ouagadougou 03, Burkina Faso. \\ *Auteur correspondant, E-mail: soulsib@yahoo.fr, Tel: +22650362664; +22670233138; \\ 09 BP 780 Ouagadougou 09, Burkina Faso.
}

\section{RESUME}

Les antinutritionnels ont été le plus souvent ignorés dans l'évaluation de la qualité des fourrages ligneux dont la part augmente continuellement dans le bol alimentaire des ruminants au Burkina Faso. L'objectif de l'étude a été de caractériser et de quantifier les coumarines dans les feuilles et/ou les fruits de 15 ligneux fourragers couramment appétés par les ruminants à Diarabakoko et Dionona. Des tests de caractérisation en tubes ont été réalisés sur les échantillons frais collectés pour détecter la présence des coumarines. Des dosages spectrophotométriques des coumarines ont été également réalisés à partir des extraits frais des échantillons. L'étude a révélé la présence des coumarines chez toutes les espèces à des teneurs oscillant respectivement entre 10,590 $\pm 0,354$ et $31,174 \pm 0,477 \mu \mathrm{g} E C / g$ d'extraits frais des feuilles et entre $1,250 \pm 0,014$ et $22,899 \pm 0,561 \mu \mathrm{g} \mathrm{EC} / \mathrm{g}$ d'extraits frais des fruits $(\mathrm{P}<0,05)$. L'analyse des variances a permis de distinguer 2 classes de teneurs en coumarines des feuilles dont la première a les plus faibles valeurs. Nos résultats constituent des critères supplémentaires pour le choix des ligneux fourragers en aménagement pastoral et en agroforesterie. Ils montrent aussi que le fourrage (feuilles et fruits) des espèces étudiées peut contribuer sans danger à la supplémentation des ruminants.

(c) 2013 International Formulae Group. All rights reserved.

Mots clés : Alimentation, antinutritionnels, fourrage, aménagement pastoral, ruminants.

\section{INTRODUCTION}

Au Burkina Faso, l'espace pastoral se rétrécit continuellement au profit des champs de cultures. Le fourrage des espèces herbacées notamment pérennes régresse sous l'influence de la variabilité climatique et du surpâturage. Le fourrage ligneux est davantage plus abondant dans le bol alimentaire des ruminants durant la saison sèche (Botoni,
2003). L'utilisation des fourrages des espèces ligneuses dans l'alimentation animale a suscité au Burkina Faso plusieurs travaux de recherches sur l'identification des ligneux fourragers et les valeurs nutritives de leur fourrage (Kaboré-Zoungrana, 1995; Zoundi et al., 2002 ; Kaboré-Zoungrana et al., 2008; Sanon et al., 2008 ; Zampaligré, 2012). La plupart de ces études concernent les matières 
sèche et organiques, les constituants chimiques (matière azotée totale, matières, fibres) l'ingestibilité du fourrage et la digestibilité des matières organiques et azotées totales, des constituants pariétaux et quelquefois sur les tanins. Mais aucune d'elles n'a encore porté sur les coumarines. Selon Dolan et al. (2010) les coumarines et spécifiquement les furocoumarines représentent une famille de constituants alimentaires naturels aux propriétés phototoxiques et photomutagènes. Elles sont l'un des facteurs antinutritionnels capables de réduire la valeur nutritive $\mathrm{du}$ fourrage potentiellement riche. Kumar et D'Mello (1995) rapportent qu'il est largement admis que la diversité et la sévérité des facteurs antinutritionnels des légumineuses tropicales sont plus grandes que celles des légumineuses des pays tempérés. Les effets dépressifs des facteurs antinutritionnels ne sont notables chez les ruminants que si la proportion du fourrage des ligneux dans les rations alimentaires est élevée (Njidda, 2010). Aussi, Dolan et al. (2010) soulignent que les furanocoumarines 5-MOP et 8-MOP provoquent en présence de rayonnement ultraviolet A des tumeurs de la peau chez les animaux de laboratoire.

La présente étude a consisté à caractériser et à quantifier les coumarines dans les feuilles et/ou les fruits de 15 ligneux fourragers afin de pallier l'une des insuffisances de connaissances dans l'utilisation du fourrage ligneux. Elle a contribué à une meilleure connaissance des plantes fourragères pour améliorer l'alimentation des ruminants.

\section{MATERIEL ET METHODES}

\section{Milieu d'étude}

La présente étude a été menée dans les villages de Diarabakoko et de Dionona. Ces deux villages contigus sont situés dans le district phytogéographique de la Comoé (Guinko, 1989) à l'Ouest du Burkina Faso, à environ $470 \mathrm{~km}$ de Ouagadougou. Le site d'étude se situe entre $10^{\circ} 30^{\prime} 09,5^{\prime}$ ' et $10^{\circ} 30^{\prime} 06,4^{\prime \prime}$ ' de latitude Nord, $4^{\circ} 43^{\prime} 55,0^{\prime}$ ' et $4^{\circ} 53^{\prime} 08,0$ ' de longitude Ouest. Le climat est de type tropical caractérisé par une saison des pluies et une saison sèche allant respectivement de mai à octobre et de novembre à avril. La pluviométrie annuelle oscille entre $1200 \mathrm{~mm}$ et $1400 \mathrm{~mm}$ (Guinko, 1984). Cependant la zone connaît une baisse de la pluviosité depuis au moins trois décennies. Ainsi, la station agrométéorologique de Bérégadougou à environ $40 \mathrm{~km}$ du site d'étude, a enregistré de 1982 à 2011 une quantité moyenne de 1032,8 mm de pluies par an. A la même période, les températures moyennes ont varié entre $21^{\circ} \mathrm{C}$ et $34,6{ }^{\circ} \mathrm{C}$ et l'humidité relative moyenne est de $62 \%$.

La végétation est une mosaïque de formations végétales constituée de forêts claires, de cordons ripicoles et de savanes (Guinko, 1984 ; Ouoba, 2006). L'ensemble des formations végétales spontanées constitue l'espace sylvo-pastoral qui se rétrécit chaque année au profit des champs de cultures et des vergers. De plus, l'augmentation des effectifs de bovins suite à l'installation des migrants peuls, les péjorations climatiques et le passage des feux annuels tardifs limitent le disponible fourrager des herbacées en saison sèche et favorisent ainsi l'augmentation de la consommation $\mathrm{du}$ fourrage des espèces ligneuses.

\section{Matériel végétal}

L'étude a porté sur les feuilles et/ou les fruits de 15 espèces spontanées réparties dans 7 familles (Tableau 1).

Les espèces ont été choisies suite à une revue bibliographique des écrits de Arbonnier (2004), Ouédraogo-Koné et al. (2006), Sanon al. (2007), Guigma et al. (2012) et à des interviews auprès des pasteurs et agropasteurs des villages concernés par notre étude. Les principaux critères ont été sur l'appétibilité, la richesse en protéines (espèces 
de la famille des Fabaceae) et la famille des espèces fourragères étudiées.

\section{Collecte des échantillons}

Un rameau feuillu a été prélevé en novembre 2012 sur trois pieds différents de chaque espèce portant des jeunes feuilles et/ou des fruits. Les fruits de Acacia sieberiana, Baissea multiflora, Cassia sieberiana et de Piliostigma thonningii étaient immatures tandis que ceux ce de Faidherbia albida et de Prosopis africana étaient mûrs et secs. Les gousses mures de $F$. albida ont été récoltées en mars 2012.

Les échantillons frais ont été étiquetés et transportés dans des sacs à jute du site d'étude au Laboratoire de Biochimie et de Chimie Appliquées de l'Université de Ouagadougou. Les sacs ont été fréquemment mouillés afin que les échantillons frais gardent leur état initial. Seuls les feuilles et les fruits non détériorés ont été analysés. Les fruits secs de $F$. albida et de $P$. africana ont été mis dans des sachets plastiques avant d'être transportés aux laboratoires.

\section{Extraction}

Les différents échantillons de plantes $(10 \mathrm{~g})$ ont été broyés ou transformés en poudre avant d'être extraits à la température ambiante avec $100 \mathrm{~mL}$ de méthanol pendant 24 heures sous agitation mécanique. Les extraits méthanoliques ainsi obtenus après filtration ont été directement utilisés pour la caractérisation des coumarines.

Pour déterminer la teneur des coumarines, 1 gramme d'échantillon frais finement broyé de chaque espèce a été extrait avec $10 \mathrm{~mL}$ de méthanol $80 \%$ pendant 10 minutes et filtré. Le filtrat ainsi obtenu a été dilué au centième (1/100).

\section{Caractérisation des coumarines}

La détection des coumarines a été réalisée par le test des coumarines. Ainsi, les différents extraits méthanoliques ont été dilués de moitié avec de l'eau distillée et répartis dans 2 tubes à essai. Le premier a servi de témoin tandis que $0,5 \mathrm{~mL}$ de solution de $\mathrm{NH}_{4} \mathrm{OH}$ titrée $10 \%$ a été ajoutée dans le deuxième tube. L'apparition d'une fluorescence bleue ou verte à la lampe UV à $365 \mathrm{~nm}$ indique la présence des coumarines (Wagner et Bladt, 1996; Ciulei, 1982).

\section{Dosage des coumarines}

Les coumarines naturelles sont des métabolites secondaires synthétisés par les feuilles des plantes. Elles sont sous forme d'hétérosides et différemment reparties dans les différentes parties d'une même plante.

La teneur en coumarines de chaque espèce fourragère a été déterminée par spectrophotométrie. Pour ce faire, la courbe de calibration de la coumarine a été tracée selon la méthode décrite par Malik et al. (2012) en utilisant la solution mère de coumarine $(100 \mu \mathrm{g} / \mathrm{mL})$ à la longueur d'onde maximale de $290 \mathrm{~nm}$. La solution mère a été obtenue en solubilisant $100 \mathrm{mg}$ de la coumarine (2H-1- benzopyran-2-one) dans 10 $\mathrm{mL}$ de méthanol $80 \%$. La solution test de concentration $10 \mu \mathrm{g} / \mathrm{mL}$ a donné des solutions de concentrations différentes après une série de dilutions dans le méthanol $80 \%$. Trois cents microlitres de cette solution test ont été utilisés pour faire le balayage dans la zone de 200 et $400 \mathrm{~nm}$. La longueur d'onde maximale obtenue se situe à $290 \mathrm{~nm}$. Ensuite, la courbe de calibration a été réalisée avec la solution mère de coumarine $(100 \mu \mathrm{g} / \mathrm{mL})$ à la longueur d'onde maximale. Les concentrations de 50 $\mu \mathrm{g} / \mathrm{mL}, 25 \mu \mathrm{g} / \mathrm{mL}, 12,50 \mu \mathrm{g} / \mathrm{mL}, 6,25 \mu \mathrm{g} / \mathrm{mL}$, $3,125 \mu \mathrm{g} / \mathrm{mL}, \quad 1.5625 \mu \mathrm{g} / \mathrm{mL}$ et 0,78185 $\mu \mathrm{g} / \mathrm{mL}$ obtenues après dilution de la solution mère ont permis de tracer la courbe étalon dont la droite d'équation est : $\mathrm{y}=0,059 \mathrm{x}+$ 833 et $\mathrm{R}^{2}=0,996$.

Les teneurs en coumarines ont été déterminées en utilisant $300 \mu \mathrm{L}$ des extraits frais pour le dosage direct à la longueur d'onde de $290 \mathrm{~nm}$. Les teneurs ont été exprimées en microgramme Equivalent Coumarines par gramme ( $\mu \mathrm{g} \mathrm{EC/g})$ d'extraits frais et pourraient être exprimées en $\mathrm{mg} / \mathrm{mL}$. 
Tableau 1 : Espèces choisies et parties prélevées.

\begin{tabular}{lll}
\hline Noms des espèces & Familles & Parties prélevées \\
\hline Sclerocarya birrea (A. Rich.) Hochst & Anacardiaceae & Feuilles \\
Baissea multiflora A. DC. & Apocynaceae & Fruits** \\
Anogeissus leiocarpa (DC.) Guil. et Per. & Combretaceae & Feuilles \\
Acacia sieberiana DC. & Fabaceae-Mimosoideae & Feuilles, Fruits** \\
Afzelia africana Sm. ex Pers. & Fabaceae-Caesalpinioideae & Feuilles \\
Cassia sieberiana DC. & Fabaceae-Caesalpinioideae & Fruits** \\
Faidherbia albida (Delile) A. Chev. & Fabaceae-Mimosoideae & Feuilles, Fruits* \\
Dichrostachys cinerea (L.) Wight et Arn. & Fabaceae-Mimosoidea & Feuilles \\
Piliostigma thonningii (Schumach.) Milne & Fabaceae-Caesalpinioideae & Fruits** \\
Pericopsis laxiflora (Benth.) Meeuwen & Fabaceae-Faboideae & Feuilles \\
Prosopis africana (Guill. et Perr.) Taub & Fabaceae-Caesalpinioideae & Feuilles, Fruits* \\
Pterocarpus erinaceus Poir. & Fabaceae-Faboideae & Feuilles \\
Khaya senegalensis (Desr.) A. Juss. & Meliaceaea & Feuilles \\
Mitragyna inermis (Willd.) Kuntze & Rubiaceae & Feuilles \\
Zanthoxylum zanthoxyloides (Lam.) Zepern. & Rutaceae & Feuilles \\
et Timler. & &
\end{tabular}

Fruits $^{*}=$ Fruits matures, Fruits $* *=$ Fruits $=$ immatures.

\section{Analyses statistiques des données}

Les teneurs moyennes des coumarines ont été calculées en utilisant le logiciel Microsoft Excel10. Le logiciel IBM SPSS Statistics 20 a servi à l'analyse de variance à un facteur pour discriminer les moyennes à $\mathrm{p}$ $<0,05$ en appliquant le test de Tukey.

\section{RESULTATS}

\section{Caractérisation des coumarines}

Les tests en tubes ont montré la présence de coumarines dans les feuilles de toutes les espèces sauf dans celles de $A$. sieberiana, Anogeissus leiocarpa et Dichrostachys cinerea, (Tableau 2). Les coumarines sont également présentes dans les fruits de A. sieberiana, B. multiflora, $C$. sieberiana, Prosopis africana (Tableau 2). Par contre, elles n'ont pas été détectées dans les gousses de $F$. albida et $P$. thonningii.

\section{Teneurs en coumarines}

Les teneurs moyennes en coumarines des feuilles et fruits des espèces étudiées sont présentées dans le Tableau 3. Les teneurs moyennes en coumarines des extraits frais des feuilles ont varié de 10,590 $\pm 0,354 \mu \mathrm{g} \mathrm{EC/g}$ (F. albida) à $31,174 \pm 0.477 \mu \mathrm{g} \mathrm{EC/g}(D$. cinerea). La comparaison des teneurs moyennes (à $\mathrm{P}<0,05)$ a permis de regrouper les concentrations des coumarines des feuilles des différents ligneux en 2 classes. Ce sont : - la $1^{\text {ère }}$ classe est constituée de $F$. albida, Khaya senegalensis, Acacia sieberiana, Zanthoxylum zanthoxyloides, Pterocarpus erinaceus, Afzelia africana et de Mitragyna inermis dont les teneurs ont été comprises entre $10,590 \pm 0,354$ et $29,160 \pm 0,271 \mu \mathrm{g} \mathrm{EC/g}$; - la $2^{\text {ème }}$ est constituée de Sclerocarya birrea, $P$. africana, $P$. laxiflora, A. leiocarpa et de D. cinerea dont les concentrations ont été comprises entre $29,277 \pm 0,175$ et $31,174 \pm 0,477 \mu \mathrm{g} \mathrm{EC} / \mathrm{g}$

- Les concentrations en coumarines des fruits ont été comprises entre 1,250 $\pm 0,014 \mu \mathrm{g} \mathrm{EC} / \mathrm{g}$, (P. africana) et $22,899 \pm 0,561 \mu \mathrm{g} \mathrm{EC/g}(P$. thonningii). 
Tableau 2 : Caractérisation des coumarines dans les organes.

\begin{tabular}{llc}
\hline Espèces & Organes & Détection \\
\hline Acacia sieberiana & Feuilles & - \\
Afzelia africana & Feuilles & + \\
Anogeissus leiocarpa & Feuilles & - \\
Dichrostachys cinerea & Feuilles & - \\
Sclerocarya birrea & Feuilles & + \\
Kaya senegalensis & Feuilles & + \\
Mitragyna inermis & Feuilles & + \\
Pericopsis laxiflora & Feuilles & + \\
Prosopis africana & Feuilles & + \\
Pterocarpus erinaceus & Feuilles & + \\
Zanthoxylum zanthoxyloides & Feuilles & + \\
Acacia sieberiana & Gousses & + \\
Baissea multiflora & Gousses & + \\
Cassia sieberiana & Gousses & + \\
Faidherbia albida & Gousses & - \\
Piliostigma thonningii & Gousses & - \\
Prosopis africana & Gousses & + \\
\hline
\end{tabular}

+: Présence -: non détecté

Tableau 3 : Teneurs moyennes en coumarines des feuilles et fruits.

\begin{tabular}{lcc}
\hline \multirow{2}{*}{ Espèces fourragères } & \multicolumn{2}{c}{ Teneur $(\boldsymbol{\mu g}$ EC/g d'extraits frais) } \\
\cline { 2 - 3 } & \multicolumn{1}{c}{ Feuilles } & Fruits \\
\hline Faidherbia albida & $10,590^{\mathrm{a}} \pm 0,354$ & $1,709^{\mathrm{a}} \pm 0,021$ \\
Khaya senegalensis & $16,750^{\mathrm{b}} \pm 2,283$ & $\mathrm{Nd}$ \\
Acacia sieberiana & $20,317^{\mathrm{b}} \pm 0,153$ & $18,397^{\mathrm{b}} \pm 0,262$ \\
Zanthoxylum zanthoxyloides & $20,317^{\mathrm{c}} \pm 1,021$ & $\mathrm{Nd}$ \\
Pterocarpus erinaceus & $25,342^{\mathrm{d} \pm 0,876}$ & $\mathrm{Nd}$ \\
Afzelia africana & $27,368^{\mathrm{d}, \mathrm{e}} \pm 0,485$ & $\mathrm{Nd}$ \\
Mitragyna inermis & $29,160^{\mathrm{e}}, \mathrm{f} \pm 0,271$ & $\mathrm{Nd}$ \\
Sclerocarya birrea & $29,277^{\mathrm{f}} \pm 0,175$ & \\
Prosopis africana & $29,972^{ \pm} \pm 0,109$ & $1,250^{\mathrm{a}} \pm 0,014$ \\
Pericopsis laxiflora & $30,740^{\mathrm{f}} \pm 0,092$ & $\mathrm{Nd}$ \\
Anogeissus leiocarpa & $31,140^{ \pm} \pm 0,042$ & $\mathrm{Nd}$ \\
Dichrostachys cinerea & $31,174^{\mathrm{f}} \pm 0,477$ & $\mathrm{Nd}$ \\
Cassia sieberiana & $\mathrm{Nd}$ & $17,679^{ } \pm 0,640$ \\
Baissea multiflora & $\mathrm{Nd}$ & $22,482^{\mathrm{c}} \pm 0,026$ \\
Piliostigma thonningii & $\mathrm{Nd}$ & $22,899^{\mathrm{c}} \pm 0,561$ \\
\hline Les moyennes d'une même colonne avec des exposants différents sont significativement différentes $(\mathrm{p}<0,05)$. \\
Nd = Non déterminé. EC $=$ équivalant coumarines.
\end{tabular}




\section{DISCUSSION}

Dans cette étude, les coumarines ont été détectées dans toutes les espèces végétales appartenant aux familles des Anacardiaceae, Apocynaceae, Combretaceae, Fabaceae, Meliaceae, Rubiaceae et Rutaceae. Nos résultats confirment et complètent ceux de Koné (2009) et de Bourgaud et al. (2006) qui montrent que les coumarines sont présentes chez les Apiaceae, Compositae, Fabaceae, Lamiaceae, Lauraceae, Rosaceae, Rubiaceae, Rutaceae et Solanaceae. Bien que la détermination des différents types de coumarines n'ait pas été l'objet de notre étude, il est scientifiquement admis qu'il existe plusieurs composés coumariniques variant en fonction des espèces végétales (Koné, 2009). Ainsi, les furocoumarines, issues de la prénylation des coumarines, se rencontrent principalement dans la famille de Rutaceae (Dolan et al., 2010). Ces travaux de Dolan et al. (2010) nous suggèrent que parmi les échantillons étudiés seules les feuilles de Z. zanthoxyloides contiendraient des flurocoumarines.

Par ailleurs, les concentrations des coumarines dans les feuilles des ligneux fourragers de la classe 1 sont significativement inférieures à celles de la classe 2. Dans les 2 classes, les différences significatives de concentrations en coumarines ne sont notées que dans la classe 1. D'une manière générale, les teneurs en coumarines varient entre les différentes espèces et familles végétales comme le rapporte Norwegian Scientific Committee for Food Safety (2010). Par contre, les stades de développement disparates des feuilles expliqueraient partiellement les différences non significatives constatées entre les concentrations en coumarines des feuilles des espèces de la classe 2, entre celles de A. sieberiana et de $Z$. zanthoxyloides, entre celles $P$. erinaceus et de A. africana de la classe 1. En effet, bien que les feuilles aient été cueillies jeunes elles n'étaient certainement pas au même stade de développement. $F$. albida et $K$. senegalensis, avec des teneurs en coumarines respectives de $10,590 \pm 0,354$ et $16,750 \pm 2,283 \mu \mathrm{g} E C / g$, sont d'intérêts particuliers en élevage des ruminants. En effet, elles gardent leurs feuilles durant toute la saison sèche (Ali et al., 2007) et font partie des espèces les plus appétées (Sanon, 2007 ; Zampaligré, 2012).

Les teneurs en coumarines des fruits immatures de A. siebériana $(18,397 \pm 0,262 \mu \mathrm{g}$ $\mathrm{EC} / \mathrm{g})$ des fruits mûrs de $F$. albida $(1,709 \pm 0,021 \mu \mathrm{g} \mathrm{EC} / \mathrm{g}) \quad$ et de P. africana $(1,250 \pm 0,014 \mu \mathrm{g} \mathrm{EC} / \mathrm{g})$ ont été inférieures respectivement à $20,317 \pm 0,153$; $10,590 \pm 0,354$ et $29,972 \pm 0,109 \mu \mathrm{g} \mathrm{EC} / \mathrm{g}$ des feuilles des mêmes espèces. Cependant, Szewczyk et Bogucka-Kocka (2012) indiquent que les coumarines sont plus abondantes dans les fruits et les racines que dans les fleurs et feuilles. Mais les faibles teneurs en coumarines observées dans les fruits mûrs et secs de $F$. albida et de $P$. africana suggèrent que les fruits s'appauvrissent en coumarines au fil du temps. Les investigations aux différents stades phénologiques permettront de mieux cerner les concentrations en coumarines des 15 ligneux étudiés.

Néanmoins, les valeurs actuelles des coumarines $(1,250 \pm 0,014$ à $31,174 \pm 0,477 \mu \mathrm{g}$ $\mathrm{EC} / \mathrm{g}$ ) sont comprises entre les concentrations de 5 à $834 \mathrm{mg} / \mathrm{ml}$ d'extraits méthanoliques auquelles Ojala et al. (2000) attribuent un rôle de protection contre les agents pathogènes de ces espèces. En effet, Masuda et al. (1998) indiquent que les coumarines sont considérées comme des phytoalexines produites en réponse aux attaques pathogènes ou des stress abiotiques. Dans la présente étude elles pourraient avoir une influence négative sur l'ingestion et la digestibilité des fourrages comme le montrent les travaux de Baumont (1996). Cet auteur en incorporant la coumarine à petite dose (non mentionnée) dans l'aliment a constaté une réduction de $32 \%$ la quantité ingérée et de 3,9\% la digestibilité in vitro contre des réductions respectives de $31 \%$ et $52 \%$ causées par l'acide tannique incorporé. Mais il indique que la coumarine n'a d'effet négatif sur la quantité ingérée que si l'animal dispose d'un seul aliment. Paradoxalement, les animaux 
développent une préférence pour la coumarine en situation de choix (Baumont, 1996). Genin (1990) rapporte que les choix alimentaires ne sont pas contrôlés par un seul facteur chimique mais plutôt sur des "compromis" entre des teneurs élevées en nutriments, comme les matières azotées et des composés répulsifs.

Par ailleurs, les teneurs en coumarines des 15 ligneux fourragers semblent faibles pour provoquer des pathologies du foie chez les ruminants aux pâturages. En effet, Burfield (2008) rapporte de l'Autorité européenne de sécurité des aliments (EFSA) que la dose maximale sans effet (NOAEL, "no observable adverse effect level") globale de la toxicité du foie chez les chiens de deux ans est de $10 \mathrm{mg}$ de coumarines par kg de poids corporel par jour. Carlton et al. (1996) et NTP (1993) relèvent également que les rats paraissent être les espèces animales les plus sensibles aux coumarines à une dose minimale entraînant un effet néfaste de l'ordre de 16 à $18 \mathrm{mg} / \mathrm{kg}$ de poids vif par jour.

\section{Conclusion}

Les 15 ligneux fourragers étudiés renferment des coumarines dans leurs feuilles et fruits. Mais, les concentrations des coumarines dans ces différentes espèces étudiées ne constituent pas un danger pour la santé des ruminants. Elles joueraient à la fois un rôle répulsif limité qui oriente les herbivores sur le choix des ligneux fourragers et un rôle dépressif sur l'ingestion volontaire et la digestibilité des fourrages des ligneux.

Les teneurs en coumarines peuvent servir de critères de choix des ligneux fourragers en aménagement pastoral et en agroforesterie. L'étude devra s'étendre à un nombre plus élevé d'espèces ligneuses fourragères, aux différents périodes des deux saisons et aux stades phénologiques. L'accent devra être mis sur la relation entre les teneurs en coumarines et celles des autres facteurs antinutritionnels pour accroître les critères de choix des ligneux fourragers dans les cadres de la supplémentation des animaux et de l'aménagement pastoral et l'agroforesterie.

\section{REMERCIEMENTS}

Les auteurs remercient le Ministère des Ressources Animales et Halieutiques à travers le Projet BKF 017 Azawak - Ressources pastorales pour avoir financé cette étude.

\section{REFERENCES}

Ali M, Saadou M, Jean L. 2007. Phénologie de quelques espèces ligneuses du Parc national «W» du Niger. Sécheresse, 1E(4): 1-13.

Arbonnier M. 2004. Arbres, Arbustes et Lianes des Zones Sèches d'Afrique de l'Ouest ( $2^{\text {ème }}$ édn). CIRAD, MNHN, UICN.

Baumont R. 1996. Palatabilité et comportement alimentaire chez les ruminants. INRA Prod. Anim., 9(5): 349358.

Botoni EH. 2003. Interactions élevagesenvironnement. Dynamique des paysages et évolution des pratiques pastorales dans les fronts pionniers du Sud-Ouest du Burkina Faso. Thèse de doctorat, Université Paul Valery-Montpellier III, France, p. 295.

Bourgaud F, Hehn A, Larbat R, Doeper S, Gontier E, Kellner S, Matern U. 2006. Biosynthesis of coumarins in plants: a major pathway still to be unraveled for cytochrome P450 enzymes. Phytochemistry Reviews, 5: 293-308.

Burfield T. 2008. Coumarin: The real story. Available online: http://www.leffingwell. com/Coumarin-story-update2.pdf (accessed on 21 July 2010).

Carlton BD, Aubrun JC, Simon GS. 1996. Effects of coumarin following perinatal and chronic exposure in Sprague-Dawley rats and CD-1 mice. Fundamental and Applied Toxicology, 30: 145-151.

Ciulei I. 1982. Practical Manuals on the Industrial Utilization of Chemical and Aromatic Plants. Methodology for analysis of vegetable drugs. Ministry of Chemical Industry, Bucharest, p.67.

Dolan LC, Matulka RA, Burdock GA. 2010. Naturally occurring food Toxins. Journal Toxins, 2: 2289-2332. 
Genin D. 1990. Composition chimique des plantes ingérées et régime saisonnier de caprins sur un parcours aride. Fourrages, 124: 385-397.

Guigma Y, Zerbo P, Millogo-Rasolodimby J. 2012. Utilisation des espèces spontanées dans trois villages contigus du Sud du Burkina Faso. Tropicultura, 30(4): 230235.

Guinko S. 1984. Végétation de la Haute Volta. Thèse de doctorat d'Etat ès Sciences Naturelles. Université de Bordeaux, Tome I, p. 318.

Guinko S. 1989. Contribution à l'étude de la végétation et de la flore du Burkina Faso: les territoires phytogéographiques. Bulletin I.F.A.N. T46 Ser. A, 1: 1984 1985.

Kaboré-Zoungrana YC. 1995. Composition chimique et valeur nutritive des herbacées et ligneux des pâturages naturels soudaniens et des sous-produits du Burkina Faso. Thèse de doctorat d'Etat ès Sc. FAST, UO, Ouagadougou, Burkina Faso, p. 201.

Kaboré-Zoungrana C, Diarra B, Adandedjan $\mathrm{C}$ et Savadogo S. 2008. Valeur nutritive de Balanites aegyptiaca pour l'alimentation des ruminants. Livestock Research for Rural Development, 20(56).

Koné D. 2009. Enquête ethnobotanique de six plantes médicinales maliennes : extraction, identification, d'alcaloïdes Caractérisation, quantification de polyphénols : étude de leur activité antioxydante. Thèse de doctorat de Chimie organique, Univ. Bamako, Mali, p. 188.

Kumar R, D’Mello JPF. 1995. Anti-nutritional factors in forage legumes. In Tropical Legumes in Animal Nutrition, D'Mello J PF, Devendra D (éds) CAB. International Wallingford: UK: 95-133.

Malik A, Kushnoor A, Saini V, Singhal S, Kumar S, Yadav YC. 2012. Analytical Method Development of Nutraceutical: Umbelliferone. Pharma Science Monitor, 3(1): 67-73.
Masuda T, Takasugi M, Anetai M. 1998. Psoralen and other linear furanocoumarins as phytoalexins in Glehnialittoralis. Phytochemistry, 47: 1316.

Njidda AA. 2010. Chemical Composition, Fiber Fraction and Anti-Nutritional Substances of Semi-arid Browse Forages of North-Eastern Nigeria. Department of Animal Science, Bayero University, Kano P.M.B. 3011, Kano State, Nigeria. Nigerian Journal of Basic and Applied Science, 18(2): 181-188.

Norwegian Scientific Committee for Food Safety. 2010. Risk assessment of coumarin intake in the Norwegian population. Opinion of the Panel on Food Additives, Flavourings, Processing Aids, Materials in Contact with Food and Cosmetics of the Norwegian Scientific Committee for Food Safety; 09/405-2 final, p. 68

NTP (National Toxicology Program), 1993. Toxicology and carcinogenesis studies of coumarin (CAS No. 91-64-5) in F344/N rats and $\mathrm{B} 6 \mathrm{C} 3 \mathrm{~F} 1$ mice (gavage studies). Technical Rep. Series No 422, NIH Publication No. 92-3153. U.S. Department of Health and Human Services, Public Health Service, National Institutes of Health, Research Triangle Park, NC.

Ouédraogo-Koné S, Kaboré-Zoungrana CY, Ledin I. 2006. Behaviour of goats, sheep and cattle on natural pasture in the subhumid zone of West Africa. Livestock Science, 105: 244-252.

Ojala T, Remes S, Haansuu P, Vuorela H, Hiltunen R, Haahtela K, Vuorela P. 2000. Antimicrobial activity of some coumarin containing herbal plants growing in Finland. Journal of Ethnopharmacology, 73: 299-305.

Ouoba P. 2006. Flore et végétation de la forêt classée de Niangologo, Sud-Ouest du Burkina Faso. Thèse de doctorat, Université de Ouagadougou, p.144.

Sanon HO, Kaboré-Zoungrana C, Ledin I. 2007. Behaviour of goats, sheep and 
cattle and their selection of browse species on natural pasture in a Sahelian area. Small Ruminant Research, 67: 6474.

Sanon HO, Kaboré-Zoungrana C, Ledin I. 2008. Nutritive value and voluntary feed intake by goats of three browse fodder species in the Sahelian zone of West Africa. Animal Feed Science and Technology, 144: 97-110.

Szewczyk K, Bogucka-Kocka A. 2012. Analytical Methods for Isolation, Separation and Identification of Selected Furanocoumarins in Plant Material. In Phytochemicals - A Global Perspective of Their Role in Nutrition and Health Venketeshwer Rao p (éd).cm; 58-92.

Wagner H, Bladt S. 1996. Plant Drug Analysis: A Thin Layer Chromatography Atlas (2nd edn). Springer-Verlag: Berlin Heidelberg.
Zampaligré N. 2012. The role of ligneous vegetation for livestock nutrition in the sub-Sahelian and Sudanian zones of West Africa: Potential effects of climate change. PhD thesis, University of Kassel, Faculty of Organic Agricultural Sciences/Animal Husbandry in Tropics and Subtropics, p. 102.

Zoundi JS, Nianogo AJ, Sawadogo L. 2002. Utilisation optimale de ressources alimentaires localement disponibles pour l'engraissement des ovins au sein des exploitations mixtes agriculture-élevage du plateau central du Burkina Faso. Revue Elev. Med. Vélo Pays trop., 55(1): 53-62. 